\title{
Interpersonal sensitivity mediates the effects of child abuse and affective temperaments on depressive symptoms in the general adult population
}

\author{
This article was published in the following Dove Press journal: \\ Neuropsychiatric Disease and Treatment \\ 5 October 2017 \\ Number of times this article has been viewed
}

\author{
Ayano Otsuka' \\ Yoshikazu Takaesu' \\ Mitsuhiko Sato' \\ Jiro Masuya' \\ Masahiko Ichiki' \\ Ichiro Kusumi ${ }^{2}$ \\ Takeshi Inoue' \\ 'Department of Psychiatry, \\ Tokyo Medical University, Tokyo, \\ ${ }^{2}$ Department of Psychiatry, Hokkaido \\ University Graduate School of \\ Medicine, Sapporo, Japan
}

\begin{abstract}
Background: Recent studies have suggested that multiple factors interact with the onset and prognosis of major depressive disorders. In this study, we investigated how child abuse, affective temperaments, and interpersonal sensitivity are interrelated, and how they affect depressive symptoms in the general adult population.
\end{abstract}

Subjects and methods: A total of 415 volunteers from the general adult population completed the Patient Health Questionnaire-9, the Temperament Evaluation of Memphis, Pisa, Paris, and San Diego-Autoquestionnaire version, the Child Abuse and Trauma Scale, and the Interpersonal Sensitivity Measure, which are all self-administered questionnaires. Data were subjected to structural equation modeling (Mplus), and single and multiple regression analyses.

Results: The effect of child abuse on depressive symptoms was mediated by interpersonal sensitivity and 4 affective temperaments, including depressive, cyclothymic, anxious, and irritable temperaments. In addition, the effect of these temperaments on depressive symptoms was mediated by interpersonal sensitivity, indicating the indirect enhancement of depressive symptoms. In contrast to these 4 temperaments, the hyperthymic temperament did not mediate the effect of child abuse on depressive symptoms; its effect was not mediated by interpersonal sensitivity. However, a greater hyperthymic temperament predicted decreased depressive symptoms and interpersonal sensitivity, independent of any mediation effect.

Limitations: Because this is a cross-sectional study, long-term prospective studies are necessary to confirm its findings. Therefore, recall bias should be considered when interpreting the results. As the subjects were adults from the general population, the results may not be generalizable towards all patients with major depression.

Conclusion: This study suggests that child abuse and affective temperaments affect depressive symptoms partly through interpersonal sensitivity. Interpersonal sensitivity may have a major role in forming the link between abuse, affective temperament, and depression.

Keywords: child abuse, affective temperament, TEMPS-A, interpersonal sensitivity, depression, structural equation modeling

\section{Introduction}

Multiple factors have been found to influence the onset of depression or depressive symptoms, such as genetic, environmental, and personality factors, as well as physical diseases. Child abuse, which is an environmental factor that includes emotional abuse, physical abuse, sexual abuse, emotional neglect, and physical neglect, increases the prevalence of depression. ${ }^{1-4}$ With respect to personality traits, depressive, cyclothymic,
Correspondence: Takeshi Inoue Department of Psychiatry, Tokyo Medical University, 6-7-I, Nishishinjuku, Shinjuku-ku, Tokyo 160-0023, Japan

Tel +8 I 333426 III

Fax +8I 333404499

Email tinoue@tokyo-med.ac.jp 
anxious, and irritable temperaments are more common in patients with unipolar depression or bipolar disorder than in healthy controls, and scores of these temperaments are correlated with depressive symptoms in adults within the general population. ${ }^{5-7}$ Furthermore, a close relationship between interpersonal sensitivity and depression has also been reported. ${ }^{8-10}$ Boyce and Parker proposed the concept of interpersonal sensitivity and developed the Interpersonal Sensitivity Measure (IPSM), a self-reported measure of interpersonal sensitivity. ${ }^{8}$ Compared to healthy controls, total IPSM scores are higher in patients with depression and in subjects with a history of depression. ${ }^{8,10}$ Boyce et al reported in their prospective study that high interpersonal sensitivity was associated with an increased risk of being depressed. This suggests the involvement of personality traits as risk factors for the onset of depression. ${ }^{11}$ High IPSM total scores are also reportedly a poor prognostic factor for depression..$^{9,12}$ Consequentially, interpersonal sensitivity is suggested to be a major factor for the onset of depression.

Depression constitutes a major public health concern since it produces a substantial economic burden and negative consequences such as suicidal behavior. ${ }^{13}$ Modern pharmacological medications have demonstrated their efficacy and potential to prevent the negative effects associated with depression. ${ }^{14}$ Rapid recognition/treatment of depression is absolutely imperative for the whole community. Child abuse, certain personality traits, and negative events during adult life affect treatment responses to antidepressants. ${ }^{15-17}$ Therefore, the identification of predictors or risk factors that are relevant to the onset, diagnosis, classification of subtypes of depression, and its progression is necessary from a therapeutic viewpoint.

In this study, we have investigated the relationship between each implicated factor and depression. Child abuse affects the formation of depressive, cyclothymic, anxious, and irritable temperaments. ${ }^{6,18}$ Neglect, among the several types of child abuse, has a potent influence on these temperaments. ${ }^{6}$ Lower level of maternal care is reported to increase interpersonal sensitivity. ${ }^{10}$ Furthermore, a close relationship between interpersonal sensitivity and other personality traits, as measured by the Temperament and Character Inventory (TCI), has also been reported. ${ }^{19,20}$ Recently, using structural equation modeling, we reported that child abuse indirectly predicts the severity of depressive symptoms in adults, and its effect is mediated by personality traits, that are affective temperaments, as evaluated by the Temperament Evaluation of Memphis, Pisa, Paris, and San Diego-Autoquestionnaire version (TEMPS-A). ${ }^{6}$ Child abuse, affective temperaments,

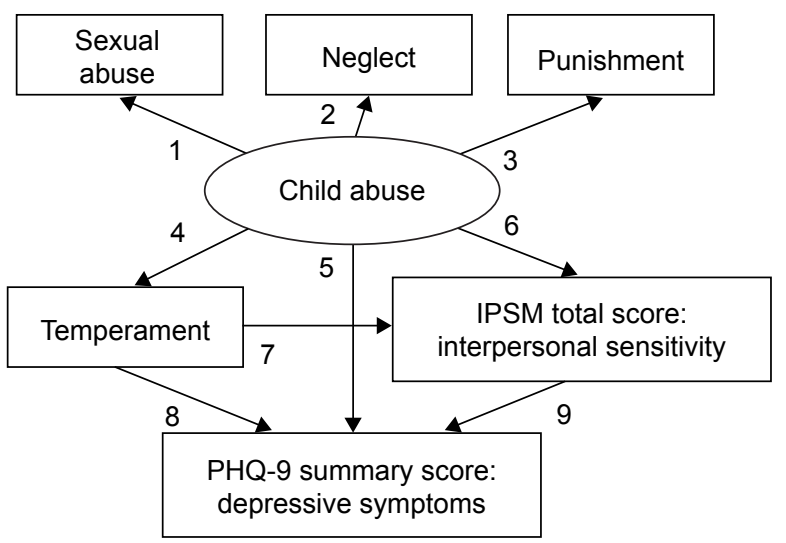

Figure I Structural equation model of the hypothesis of this study. In this model, child abuse, each temperament subscale ( 5 temperaments in 5 models) of the TEMPS-A, and interpersonal sensitivity (IPSM total score) predicted depressive symptoms (PHQ-9) in general adults. Rectangles indicate observed variables, whereas oval indicates latent variable. The standardized path coefficients $(-I$ to $+I)$ of path numbers beside the arrows are shown in Table 3 . In addition, Table 3 shows goodness-of-fit indices (CFI and RMSEA) of each model, the square sum of the multiple correlation coefficients $\left(R^{2}\right)$ of each model, and indirect effects.

Abbreviations: TEMPS-A, Temperament Evaluation of Memphis, Pisa, Paris, and San Diego-Autoquestionnaire version; IPSM, Interpersonal Sensitivity Measure; PHQ-9, Patient Health Questionnaire-9; CFI, comparative fit index; RMSEA, root mean square error of approximation.

and negative adult life events interact with each other to increase or decrease depressive symptoms. ${ }^{7}$ In this way, environmental and personality factors, including affective temperaments and interpersonal sensitivity, are directly related to the severity of depressive symptoms and may interact with each other. However, there has been no study examining the interactions among interpersonal sensitivity and other factors related to depressive symptoms or depression.

This study examined how factors that predict depression, such as child abuse, interpersonal sensitivity, and affective temperaments (depressive, cyclothymic, anxious, irritable, and hyperthymic), are related to the severity of depressive symptoms in adults within the general population. We hypothesized that child abuse influences affective temperaments, as proposed by Akiskal et al (model 1, depressive; model 2, cyclothymic; model 3, anxious; model 4, irritable; model 5, hyperthymic), ${ }^{21}$ and interpersonal sensitivity and that child abuse influences the severity of depressive symptoms directly and indirectly through these personality traits (Figure 1). We verified this hypothesis using structural equation modeling.

\section{Subjects and methods Subjects}

We distributed questionnaires to 853 volunteers, recruited by flyers or word of mouth, as candidates to participate in the study. This study was part of a larger study conducted between January 2014 and August 2014 on Japanese 
volunteers from the general adult population. ${ }^{22}$ Candidates were asked to participate and answer self-administered questionnaires. Of 853 candidates, 415 subjects (48.7\%) agreed to participate in this study and provided complete responses to the questionnaires. We did not use any exclusion criteria because we focused on the general population, not just the healthy population. Four questionnaires and a demographic questionnaire were distributed and returned anonymously. All subjects gave written, informed consent after receiving the following information: 1) the candidate's participation in this research is entirely voluntary; 2) if the candidate decides not to participate, he/she does not receive any disadvantage; and 3) the information that we collect from this research project will be kept completely confidential. This study was approved by the ethics committees of Hokkaido University Hospital and Tokyo Medical University.

\section{Questionnaires}

\section{Patient Health Questionnaire-9}

The Patient Health Questionnaire-9 (PHQ-9) is a selfadministered depression-rating scale consisting of 9 items related to the diagnostic criteria of a major depressive episode. The Japanese version was developed by Muramatsu et al. ${ }^{23}$ The PHQ-9 is answered using a 4-point scale (0-3) for symptoms that may have appeared in the past 2 weeks. The sum of the 9 items is the PHQ-9 summary score.

\section{Temperament Evaluation of Memphis, Pisa, Paris, and San Diego-Autoquestionnaire version}

The TEMPS-A measures depressive, cyclothymic, anxious, irritable, and hyperthymic temperaments quantitatively. It is a self-administered questionnaire to assess affective temperaments or traits related to mood disorders. ${ }^{21}$ The Japanese version was developed by Akiyama et al, ${ }^{24}$ and its reliability and validity have been verified. ${ }^{5}$ The TEMPS-A consists of 109 items for men and 110 items for women: depressive (21 items), cyclothymic (21 items), hyperthymic (21 items), irritable (male 20 items, women 21 items), and anxious (26 items). The TEMPS-A is answered using a 2 -point scale ("yes" is scored 2 points and "no" is scored 1 point). In our study, the average scores for each temperament were calculated, since the number of items is different for irritable temperament between men and women.

\section{Child Abuse and Trauma Scale}

The Child Abuse and Trauma Scale (CATS) is a selfadministered questionnaire in which participants retrospectively rate how frequently an abusive experience occurred during their childhood and adolescence. ${ }^{25}$ The Japanese version was developed by Tanabe et al. ${ }^{26}$ The CATS consists of 38 items and the following subscales: sexual abuse (6 items), neglect or negative home atmosphere (14 items), punishment (6 items), and other (12 items). A 5-point Likert scale (0-4; $0=$ never; $4=$ always) is used for each item. We calculated the average score of all 38 items and the items in each subscale.

\section{Interpersonal Sensitivity Measure}

The IPSM is a self-administered questionnaire that measures interpersonal hypersensitivity, which has been proposed as a possible premorbid personality for depression. ${ }^{8}$ The Japanese version was developed by Kuwabara et al, and its reliability and validity have been verified. ${ }^{27}$ The IPSM consists of 36 items. The 5 subscales of the IPSM are interpersonal awareness ( 7 items), need for approval ( 8 items), separation anxiety ( 8 items), timidity ( 8 items), and fragile inner-self (5 items). A 4-point Likert scale (1-4) is used for each item.

\section{Data analysis}

We used the scores of the PHQ-9, CATS, IPSM, and 5 temperaments of the TEMPS-A in the structural equation model. For the CATS, the latent variable "child abuse" was composed of the 3 subscales, including subscales of sexual abuse, neglect or negative home atmosphere, and punishment, as observed variables. The model was analyzed by covariance structure analysis with maximum likelihood estimation, with robust standard errors using Mplus version 7.3 (Muthén \& Muthén, Los Angeles, CA, USA). Covariance structure analysis is a statistical method for identifying causal relationships between latent or observed variables by introducing latent variables that cannot be observed directly. In case of covariance structure analysis, there are no absolute criteria for judging the suitability of the model. The models were evaluated using 2 goodness-of-fit indices: the comparative fit index (CFI) and the root mean square error of approximation (RMSEA). A good fit was indicated when CFI was 0.97 or more, and RMSEA was 0.05 or less; an acceptable fit was indicated when CFI was 0.95 or more, and RMSEA was 0.08 or less. ${ }^{28}$ All coefficients of the covariance structure analysis were standardized and are shown ( -1 to 1$)$.

In addition, Spearman's rank correlation coefficient $(\rho)$ and Mann-Whitney $U$-tests were conducted using SPSS Statistics 22 (SPSS, Chicago, IL, USA) for calculating correlations between variables and comparing variables between 2 categories (eg, male vs female), respectively.

The level of statistical significance was set at $P<0.05$. 


\section{Results}

Demographic characteristics and the CATS, TEMPS-A, IPSM, and PHQ-9 scores of subjects

The demographic characteristics and questionnaire data from 415 subjects are shown in Table 1 . We analyzed the effects of demographic categories (eg, sex) on PHQ-9 summary

Table I Characteristics, PHQ-9, CATS, TEMPS-A, and IPSM and correlation with PHQ-9 or effects on PHQ-9 in 4 I 5 general adult subjects

\begin{tabular}{|c|c|c|}
\hline $\begin{array}{l}\text { Characteristics or } \\
\text { measures }\end{array}$ & $\begin{array}{l}\text { Value } \\
\text { (number or } \\
\text { mean } \pm S D \text { ) }\end{array}$ & $\begin{array}{l}\text { Correlation with PHQ-9 } \\
(\rho) \text { or effect on PHQ-9 } \\
\text { (mean } \pm \text { SD of PHQ-9 } \\
\text { scores, U-test) }\end{array}$ \\
\hline Age & $42.3 \pm 12.0$ & $\rho=-0.110 *$ \\
\hline Sex (male:female) & $222: 193$ & $\begin{array}{l}\text { Male } 2.9 \pm 3.6 \text { vs female } \\
3.9 \pm 4.4^{*}(U \text {-test) }\end{array}$ \\
\hline Education (years) & $|5| \pm 2.0$. & $\rho=-0.056$, n.s. \\
\hline $\begin{array}{l}\text { Employment status } \\
\text { (employed:unemployed) }\end{array}$ & $352: 56$ & $\begin{array}{l}\text { Employed } 3.3 \pm 4.0 \text { vs } \\
\text { unemployed } 3.6 \pm 4.4 \text {, n.s. } \\
\text { (U-test) }\end{array}$ \\
\hline $\begin{array}{l}\text { Marital status } \\
\text { (married:unmarried) }\end{array}$ & $291: 121$ & $\begin{array}{l}\text { Married } 3.0 \pm 3.7 \text { vs } \\
\text { unmarried } 4.1 \pm 4.6^{* *} \text { (U-test) }\end{array}$ \\
\hline $\begin{array}{l}\text { Presence of cohabiter } \\
\text { (yes:no) }\end{array}$ & $302: 105$ & $\begin{array}{l}\text { Yes }=3.2 \pm 3.9 \text { vs no }=3.6 \pm 4 . \mathrm{I} \text {, } \\
\text { n.s. (U-test) }\end{array}$ \\
\hline $\begin{array}{l}\text { Presence of offspring } \\
\text { (yes:no) }\end{array}$ & $276: 136$ & $\begin{array}{l}\text { Yes }=2.9 \pm 3.9 \text { vs } \\
\text { no }=4.0 \pm 4.2^{* *}(U \text {-test })\end{array}$ \\
\hline $\begin{array}{l}\text { Comorbidity of physical } \\
\text { disease (yes:no) }\end{array}$ & $85: 326$ & $\begin{array}{l}\text { Yes }=3.8 \pm 4 . I \text { vs no }=3 . I \pm 3.9 \text {, } \\
\text { n.s. }(U \text {-test) }\end{array}$ \\
\hline PHQ-9 summary score & $3.3 \pm 3.9$ & \\
\hline \multicolumn{3}{|l|}{ CATS (average score) } \\
\hline Sexual abuse & $0.04 \pm 0.21$ & $\rho=0.164 * *$ \\
\hline Neglect & $0.62 \pm 0.58$ & $\rho=0.332 * *$ \\
\hline Punishment & $1.42 \pm 0.62$ & $\rho=0.205^{* *}$ \\
\hline Total & $0.72 \pm 1.32$ & $\rho=0.325 * *$ \\
\hline \multicolumn{3}{|c|}{ TEMPS-A (average score) } \\
\hline Depressive & $1.37 \pm 0.16$ & $\rho=0.423 * *$ \\
\hline Cyclothymic & $1.20 \pm 0.18$ & $\rho=0.50$ I** \\
\hline Hyperthymic & $1.27 \pm 0.18$ & $\rho=-0.169 * *$ \\
\hline Anxious & $1.19 \pm 0.19$ & $\rho=0.5 \mid 6^{* *}$ \\
\hline Irritable & $1.14 \pm 0.15$ & $\rho=0.360 * *$ \\
\hline \multicolumn{3}{|l|}{ IPSM } \\
\hline $\begin{array}{l}\text { Interpersonal } \\
\text { awareness }\end{array}$ & $16.72 \pm 4.04$ & $\rho=0.428^{* *}$ \\
\hline Need for approval & $21.89 \pm 3.29$ & $\rho=0.203^{* *}$ \\
\hline Separation anxiety & $17.02 \pm 4.14$ & $\rho=0.393 * *$ \\
\hline Timidity & $19.41 \pm 3.42$ & $\rho=0.362^{* *}$ \\
\hline Fragile inner-self & $9.68 \pm 5.79$ & $\rho=0.368 * *$ \\
\hline Total score & $84.24 \pm 14.95$ & $\rho=0.429 * *$ \\
\hline
\end{tabular}

Notes: Data are presented as mean \pm SD or number. $\rho=$ Spearman's rank correlation coefficient. $* P<0.05$. $* * P<0.01$.

Abbreviations: PHQ-9, Patient Health Questionnaire-9; CATS, Child Abuse and Trauma Scale; TEMPS-A, Temperament Evaluation of Memphis, Pisa, Paris, and San Diego-Autoquestionnaire version; IPSM, Interpersonal Sensitivity Measure; n.s., not significant. scores and the correlations between demographic variables, questionnaire scores, and PHQ-9 summary scores. Age, sex, marital status, and the presence of offspring significantly affected PHQ-9 summary scores. Years of education, employment status, the presence of cohabiters, and comorbidity of physical diseases did not influence PHQ-9 summary scores. The mean \pm SD of the PHQ-9 summary scores in the surveyed subjects was $3.3 \pm 3.9$, which was below the symptomatic cutoff and ranged from no symptoms to mild symptoms in most cases. ${ }^{29}$ All sub-scores of the CATS, TEMPS-A, and the IPSM were significantly correlated with PHQ-9 summary scores.

\section{Correlations between the IPSM total score, the average score on each temperament subscale of the TEMPS-A, and the average score of each subscale of the CATS}

Table 2 shows the correlations between the total IPSM score, the average scores of the temperament subscales of the TEMPS-A, and the average scores of the abuse subscales of the CATS. As shown in Table 2, the total IPSM scores were significantly and positively correlated only with neglect scores among the subscales of the CATS. The total score on the IPSM was also significantly and positively correlated with the 4 temperament scores of the TEMPS-A - depressive, cyclothymic, anxious, and irritable. In contrast, it was significantly but negatively correlated with the hyperthymic temperament score. Furthermore, there were significant and positive correlations between the 2 subscales, neglect and punishment, of the CATS and 4 temperament subscales of the TEMPS-A; however, this was not the case for the hyperthymic subscale. Sexual abuse was positively correlated only with the cyclothymic temperament among the TEMPS-A temperament subscales.

\section{Covariance structure analyses of models $\mathrm{I}-4$ in general adult subjects}

Table 3 shows the standardized coefficients of each path, goodness-of-fit indices (CFI and RMSEA) of each model, and the square sum of the multiple correlation coefficients $\left(R^{2}\right)$ of each model. The goodness of fit of models 1-4 was acceptable based on the CFI and RMSEA. Among the standardized coefficients of observed variables from the latent variable "child abuse" (Table 3), neglect showed the largest effect; although, the differences in the degree 
Table 2 Correlation $(\rho)$ between the IPSM total score, temperament subscale scores of the TEMPS-A, and subscale scores of the CATS

\begin{tabular}{|c|c|c|c|c|c|c|c|c|c|}
\hline & Neg & Pun & Sex & IPSM & Dep & Cyc & Hyp & Anx & Irr \\
\hline Neg & 1.000 & $0.308^{* *}$ & $0.222^{* *}$ & $0.337 * *$ & $0.272^{\text {** }}$ & $0.362^{* *}$ & 0.019 & $0.333^{* *}$ & $0.315^{* *}$ \\
\hline Pun & & 1.000 & $0.162^{* *}$ & 0.052 & $0.180^{* *}$ & $0.172 * *$ & -0.015 & $0.185 * *$ & $0.124 *$ \\
\hline Sex & & & 1.000 & 0.068 & 0.061 & $0.110^{*}$ & 0.016 & 0.096 & 0.078 \\
\hline IPSM & & & & 1.000 & $0.39 I^{* *}$ & 0.408 ** & $-0.097^{*}$ & $0.498 * *$ & $0.336 * *$ \\
\hline Dep & & & & & 1.000 & $0.430 * *$ & $-0.262^{* *}$ & $0.521^{* *}$ & $0.286 * *$ \\
\hline Сус & & & & & & 1.000 & 0.081 & $0.529 * *$ & $0.579 * *$ \\
\hline Нур & & & & & & & 1.000 & -0.095 & 0.086 \\
\hline Anx & & & & & & & & 1.000 & $0.497 * *$ \\
\hline Irr & & & & & & & & & 1.000 \\
\hline
\end{tabular}

Notes: $\rho=$ Spearman's rank correlation coefficient. $* P<0.05$. $* * P<0.01$.

Abbreviations: IPSM, Interpersonal Sensitivity Measure; TEMPS-A, Temperament Evaluation of Memphis, Pisa, Paris, and San Diego-Autoquestionnaire version; CATS, Child Abuse and Trauma Scale; Neg, neglect subscale; Pun, punishment subscale; Sex, sexual abuse subscale; Dep, depressive temperament; Cyc, cyclothymic temperament; Hyp, hyperthymic temperament; Anx, anxious temperament; Irr, irritable temperament.

between the CATS subscales could not be compared using the Mplus software.

Child abuse predicted significantly increased scores on 4 temperament scales - depressive, cyclothymic, anxious, and irritable - in structural equation models 1-4 (path 4 in Figure 1 and Table 3). These temperaments were related to significantly increased PHQ-9 summary scores, indicating increased depressive symptoms (path 8 in Figure 1 and Table 3). On the other hand, child abuse was also related to an increased IPSM total score, indicating increased interpersonal sensitivity (path 6 in Figure 1 and Table 3), which, in turn, increased PHQ-9 summary scores (path 9 in Figure 1 and Table 3). Child abuse was related to increased PHQ-9 summary scores directly as well (path 5 in Figure 1 and Table 3). In addition, all 4 temperament scores were related to an increased IPSM total score (path 7 in Figure 1 and Table 3).

The indirect effects of combined paths 4 and 8 (from child abuse to PHQ-9 mediated by temperament) were statistically significant in all 4 models (Table 3 ). This suggested that these 4 temperaments mediate the effect of child abuse on depressive symptoms in general adults. In other words,

Table 3 Standardized coefficients ( $-I$ to I) of paths I-9 in Figure I and indices of goodness of fit and square sums of multiple correlation coefficient $\left(R^{2}\right)$ in models I-5

\begin{tabular}{|c|c|c|c|c|c|}
\hline & Model I & Model 2 & Model 3 & Model 4 & Model 5 \\
\hline & Depressive & Cyclothymic & Anxious & Irritable & Hyperthymic \\
\hline \multicolumn{6}{|l|}{ Path } \\
\hline I. Child abuse $\rightarrow$ sexual abuse & $0.313^{* * *}$ & $0.322 * * *$ & $0.319 * * *$ & $0.324 * * *$ & $0.308 * * *$ \\
\hline 2. Child abuse $\rightarrow$ neglect & $\mathrm{I} .034 * * *$ & $1.004 * * *$ & $1.015 * * *$ & $0.998 * * *$ & $1.050 * * *$ \\
\hline 3. Child abuse $\rightarrow$ punishment & $0.404 * * *$ & $0.417 * * *$ & $0.413^{* * *}$ & $0.420 * * *$ & $0.398 * * *$ \\
\hline 4. Child abuse $\rightarrow$ temperament & $0.296 * * *$ & $0.407^{* * *}$ & $0.388 * * *$ & $0.407^{* * *}$ & 0.006 \\
\hline 5. Child abuse $\rightarrow \mathrm{PHQ}-9$ & $0.223 * *$ & $0.17 \mid * *$ & $0.162 * *$ & $0.218^{* *}$ & $0.280 * * *$ \\
\hline 6. Child abuse $\rightarrow$ IPSM & $0.197 * * *$ & $0.165^{* *}$ & $0.132 * *$ & $0.183 * *$ & $0.290 * * *$ \\
\hline 7. Temperament $\rightarrow$ IPSM & $0.319 * * *$ & $0.320 * * *$ & $0.419 * * *$ & $0.278 * * *$ & $-0.097 *$ \\
\hline 8. Temperament $\rightarrow$ PHQ-9 & $0.282^{* * * *}$ & $0.378 * * *$ & $0.460 * * *$ & $0.232^{* * *}$ & $-0.149 * * *$ \\
\hline 9. IPSM $\rightarrow$ PHQ-9 & $0.207^{* * *}$ & $0.182^{* * *}$ & $0.115^{*}$ & $0.232^{* * *}$ & $0.284 * * *$ \\
\hline Indirect effect of paths 4 and 8 (mediation by temperament) & $0.083 * * *$ & $0.154 * * *$ & $0.178 * * *$ & $0.094^{* * *}$ & -0.001 \\
\hline Indirect effect of paths 6 and 9 (mediation by IPSM) & $0.04 I^{*}$ & $0.030 *$ & 0.015 & $0.043^{*}$ & $0.082 * * *$ \\
\hline Indirect effect of paths 4 and 7 (mediation by temperament) & $0.094 * * *$ & $0.130 * * *$ & $0.163^{* * *}$ & $0.113^{* * *}$ & -0.001 \\
\hline Indirect effect of paths 4,7 , and 9 (mediation by temperament and IPSM) & $0.020 * *$ & $0.024 * *$ & $0.019^{*}$ & $0.026 * *$ & 0.000 \\
\hline Indirect effect of paths 7 and 9 (mediation by IPSM) & $0.066 * * *$ & $0.058 * *$ & $0.048^{*}$ & $0.065^{* * *}$ & -0.027 \\
\hline $\mathrm{CFI}$ & 0.971 & 0.978 & 0.986 & 0.983 & 0.975 \\
\hline RMSEA & 0.060 & 0.053 & 0.044 & 0.044 & 0.048 \\
\hline$R^{2}$ & 0.281 & 0.329 & 0.369 & 0.264 & 0.234 \\
\hline
\end{tabular}

Notes: $R^{2}=$ square sums of multiple correlation coefficient. $* P<0.05$. $* * P<0.01$. $* * * P<0.001$.

Abbreviations: PHQ-9, Patient Health Questionnaire-9; IPSM, Interpersonal Sensitivity Measure; CFI, comparative fit index; RMSEA, root mean square error of approximation. 
child abuse not only directly increased depressive symptoms but also indirectly increased depressive symptoms through increasing scores on temperament scales.

The indirect effects of combined paths 6 and 9 (from child abuse to PHQ-9 mediated by IPSM) were statistically significant in 3 of 4 models (excluding model 3, anxious temperament) (Table 3 ). This suggested that child abuse indirectly increased depressive symptoms through increasing interpersonal sensitivity.

The indirect effects of combined paths 4, 7, and 9 (from child abuse to PHQ-9 via temperament and IPSM, mediated by both temperament and IPSM) were also statistically significant in all 4 models (Table 3 ). This indicated that child abuse increased interpersonal sensitivity through increasing depressive, cyclothymic, anxious, and irritable temperaments, and this increased interpersonal sensitivity-mediated depressive symptoms.

The indirect effects of combined paths 4 and 7 (from child abuse to IPSM mediated by temperament) were statistically significant in all 4 models. This suggested that child abuse indirectly increased IPSM total scores through increasing depressive, cyclothymic, anxious, and irritable temperaments.

The indirect effects of combined paths 7 and 9 (from temperament to PHQ-9 mediated by IPSM) were statistically significant in all 4 models (Table 3 ). This suggested that depressive, cyclothymic, anxious, and irritable temperaments indirectly increased depressive symptoms through increasing interpersonal sensitivity.

In models $1-4$, the square sums of the multiple correlation coefficients $\left(R^{2}\right)$ explained $26.4 \%-37 \%$ of the variability in PHQ-9 summary scores.

We further tested structural equation modeling for subscales of the IPSM (data not shown). Interpersonal awareness and separation anxiety showed the mediation effect on the effects of child abuse and affective temperaments (depressive, cyclothymic, anxious, and irritable) in causing depressive symptoms, similar to the IPSM total score. However, other subscales of the IPSM showed different effects: need for approval did not mediate the effects of child abuse and affective temperaments; timidity showed various mediation effects on the effects of child abuse and affective temperaments; and fragile inner-self mediated the effects of child abuse, although, not affective temperaments.

\section{Covariance structure analysis of model 5 in general adult subjects}

As shown above, structural equation models 1-4 demonstrated quite similar effects. However, the effects of parameters in model 5 were very different from those seen in other models. Therefore, the results of model 5 are described separately.

The goodness of fit of model 5 was acceptable based on the CFI and RMSEA (Table 3). Among the standardized coefficients of observed variables from the latent variable, "child abuse", neglect had the largest effect (Table 3 ).

Child abuse did not influence hyperthymic temperament (path 4 in Figure 1 and Table 3), consistent with the correlational data in Table 2, where neither neglect, and punishment, nor sexual abuse was significantly correlated with hyperthymic temperament. Similar to models $1-4$, the effect of child abuse on IPSM total scores and PHQ-9 summary scores (paths 5 and 6 in Figure 1 and Table 3, respectively) and the effect of IPSM total scores on PHQ-9 summary scores (path 9 in Figure 1 and Table 3) were statistically significant. However, different from models $1-4$, hyperthymic temperament scores were related to significantly decreased IPSM total scores and PHQ-9 summary scores (paths 7 and 8 in Figure 1 and Table 3). This suggested that hyperthymic temperament decreased interpersonal sensitivity and depressive symptoms in general adults (an anti-depressogenic effect).

Among the 5 indirect effects shown in Table 3, only the indirect effect of combined paths 6 and 9 (from child abuse to PHQ-9 mediated by IPSM) was statistically significant. Accordingly, although directly related to decreased interpersonal sensitivity and depressive symptoms in general adults, the hyperthymic temperament was independent of the effect of child abuse, and thus did not mediate the effect of child abuse.

In model 5, the square sum of the multiple correlation coefficients $\left(R^{2}\right)$ explained $23.4 \%$ of the variability in PHQ-9 summary scores.

We tested structural equation modeling for the subscales of the IPSM (data not shown). In model 5, the effects of all subscales of the IPSM were similar to those of the IPSM total score.

\section{Discussion}

In this study, we found that child abuse increases the severity of depressive symptoms in general adults, directly and indirectly, through 4 affective temperaments - depressive, cyclothymic, anxious, and irritable. This result is consistent with our previous findings. ${ }^{6}$ The effect of child abuse on depressive symptoms is also mediated by interpersonal sensitivity, which has been reported for the first time in the present study. In addition, we found that 4 affective temperaments increase due to child abuse and increase interpersonal sensitivity, leading to an indirect enhancement of depressive 
symptoms. On the other hand, independent of child abuse, hyperthymic temperament scores are directly related to reduced depressive symptoms and interpersonal sensitivity. However, the effect of hyperthymic temperament on depressive symptoms is not mediated by interpersonal sensitivity, and hyperthymic temperament does not mediate any effect of child abuse. Therefore, this study suggests that hyperthymic temperament and the other 4 affective temperaments have completely different functional roles in relation to child abuse and interpersonal sensitivity. Consistent with this idea, we previously examined the interaction between affective temperaments evaluated by the TEMPS-A and child abuse or adult stress. Additionally, we demonstrated the difference in the interactive effect on depressive symptoms between the hyperthymic temperament and the other 4 temperaments of the TEMPS-A. ${ }^{7}$ Furthermore, earlier studies reported that hyperthymic temperament is positively correlated with self-directedness and sensation seeking and negatively correlated with harm avoidance. This is in contrast to the other 4 temperaments, indicating the functional importance and resilient nature of hyperthymic temperament. ${ }^{30-32}$ To our knowledge, this study is the first to clarify that interpersonal sensitivity mediates the influence of child abuse and affective temperaments on depressive symptoms, using covariance structure analysis.

No study, concerning adults from the general population, has examined the relationship between the CATS subscales and IPSM total scores. However, child abuse in case of women, seen in primary care practices, was reportedly related to increased interpersonal sensitivity. ${ }^{33}$ In past studies concerning general adults, low maternal care quality, assessed by the Parental Bonding Instrument (PBI), and high interpersonal sensitivity, assessed by the IPSM, independently increased the risk of depression. ${ }^{10}$ In addition, Otani et al examined the relationship between the IPSM and PBI scores in general adults and reported that low paternal care in men and low maternal care in women increased interpersonal sensitivity. ${ }^{20}$ Likewise, Suzuki et al also investigated the relationship between PBI scores and IPSM scores in general adults and reported that low paternal care, paternal overprotection, and maternal overprotection increased interpersonal sensitivity. ${ }^{34}$ On the other hand, Boyce et al ${ }^{35}$ and Luty et al ${ }^{19}$ reported negative results about the relationship between the quality of parenting and interpersonal sensitivity assessed by the IPSM in pregnant women and depressed patients, respectively. Because IPSM total scores were significantly correlated only with neglect, and not with punishment or sexual abuse as shown in this study, and neglect is moderately correlated with low parenting care (our unpublished data), low parenting care can be considered quite similar to neglect. Accordingly, these previous studies partly support a positive correlation between child abuse, especially neglect, and interpersonal hypersensitivity in general adults as presented in this study.

To our knowledge, there has never been any research concerning general adults, directly showing the relationship between IPSM and TEMPS-A scores. However, Perugi et al have previously examined the correlation between IPSM and TEMPS-A scores in patients with bipolar I disorder. They reported that depressive, cyclothymic, and irritable temperaments are positively correlated with IPSM total scores; however, hyperthymic temperament is negatively correlated with IPSM total scores. ${ }^{36}$ Although survey targets in their study and our studies were different, their results are largely consistent with the results of this study. Akiskal et al examined the correlation between the TCI, a famous personality autoquestionnaire, and TEMPS-A subscales in general adults. ${ }^{30}$ Depressive, cyclothymic, irritable, and anxious temperaments from the TEMPS-A were negatively correlated with selfdirectedness and cooperativeness, and positively correlated with harm avoidance; in contrast, hyperthymic temperament was positively correlated with self-directedness and negatively correlated with harm avoidance. ${ }^{30}$ Furthermore, Otani et $\mathrm{a}^{20}$ and Luty et $\mathrm{al}^{19}$ examined the correlation between the TCI subscales and IPSM total score in healthy subjects and depressed patients, respectively, and reported that the IPSM total score was negatively correlated with self-directedness and positively correlated with harm avoidance. The relationship between the IPSM and TCI and the relationship between the TCI and TEMPS-A shown in previous studies indirectly supports the validity of the correlation between the IPSM total score and the TEMPS-A subscales in general adults, as tested in this study.

A previous study reported that the presence of child abuse in psychiatric inpatients is strongly associated with highly irritable temperament, although not with hyperthymic temperament. ${ }^{18}$ On the other hand, our earlier research revealed that child abuse increased depressive symptoms indirectly through increasing depressive, cyclothymic, anxious, and irritable temperaments in general adults. ${ }^{6}$ The present study confirmed, in a general adult population, that child abuse increases depressive symptoms indirectly through depressive, cyclothymic, anxious, and irritable temperaments, although not through a hyperthymic temperament. Furthermore, interestingly, the covariance structure analyses in this study demonstrate that the mediation of the effect of child abuse on depressive symptoms by the 4 affective temperaments was further mediated by interpersonal sensitivity 
evaluated by the IPSM; however, this mediator effect via interpersonal sensitivity had nothing to do with a hyperthymic temperament.

Based on the results of this study, psychotherapy focused on interpersonal sensitivity would be useful for ameliorating depressive symptoms and targeting psychopathology for the treatment of patients who may have experienced child abuse in the past and have developed depression or depressive symptoms as a result. For example, Interpersonal Psychotherapy (IPT) is a time-limited, interpersonally focused, psychodynamically informed psychotherapy with the goal of symptom relief and improving interpersonal functioning. ${ }^{37}$ One of the IPT problem areas is interpersonal sensitivity (interpersonal deficits). While the true nature of interpersonal sensitivity is a pervasive process ingrained in the patient, IPT seeks to intervene by helping the patient resolve a current interpersonal stressor. ${ }^{37}$ The results of this study suggest that one can use the IPSM, a very easy-to-use autoquestionnaire, for the assessment of treatment response to psychotherapy and pharmacotherapy; in other words, reducing interpersonal sensitivity, which may regulate the mediation effect on the effects of child abuse and affective temperaments, can be one of the objectives or goals of psychiatric and psychological interventions.

\section{Limitations}

Because this is a cross-sectional study, a long-term prospective study is needed to confirm child abuse by objective surveys, interpersonal sensitivity in adulthood using the IPSM, and finally the onset of depression. In addition, recall bias should be considered when interpreting the results because this study assessed child abuse using a questionnaire based on the participants' memories. Depressive symptoms may have influenced the evalution of child abuse. Indeed, the scores of child abuse were significantly correlated with the severity of depressive symptoms in this study. On the other hand, an earlier review suggested a certain level of accuracy of memories in early experiences, ${ }^{38}$ and our previous study showed that the CATS scores were less influenced by the severity of depressive symptoms on the test day. ${ }^{39}$ However, this limitation urges us to conduct future prospective studies. Furthermore, because this study targeted general adults, there is the possibility that this result cannot be generalized to patients with major depression. Because female subjects reported slightly higher depressive symptoms than male subjects, the path coefficients might be different between male and female subjects. However, effects of the grouping variable (ie, sex difference) could not be analyzed due to the low goodness-of-fit indices (data not shown). In future, a sex difference would be examined in our structural equation models.

\section{Conclusion}

This study showed that child abuse indirectly strengthens interpersonal sensitivity through the increment of 4 affective temperaments - depressive, cyclothymic, anxious, and irritable - and these temperaments indirectly increase depressive symptoms through increased interpersonal sensitivity. On the other hand, independent of child abuse, hyperthymic temperament directly reduces depressive symptoms and interpersonal sensitivity. The finding that interpersonal sensitivity mediates the influence of child abuse and affective temperaments on depressive symptoms has never been reported until now. This study is the first report on this issue. Our study suggests that psychotherapy focusing on interpersonal sensitivity may be clinically useful for the treatment of depressed patients or clients with a history of child abuse.

\section{Acknowledgment}

This work was partly supported by a Grant-in-Aid for Scientific Research (No 16K10194, T Inoue) from the Japanese Ministry of Education, Culture, Sports, Science and Technology.

\section{Author contributions}

Dr Inoue and Dr Takaesu designed the study and wrote the protocol. Dr Otsuka collected and analyzed the data. All authors contributed to data analysis, drafting and critically revising the paper. In addition, all authors gave the final approval for the desired version to be published, and agreed to be accountable for all aspects of the work.

\section{Disclosure}

Yoshikazu Takaesu has received personal compensation from Otsuka Pharmaceutical, Meiji Seika Pharma, Eli Lilly, Eisai, Mitsubishi Tanabe Pharma, and Yoshitomiyakuhin and grants from Otsuka Pharmaceutical, Meiji Seika Pharma, and Eisai. Jiro Masuya has received personal compensation from Otsuka Pharmaceutical, Eli Lilly, Astellas, and Meiji Yasuda Mental Health Foundation and grants from Pfizer. Masahiko Ichiki has received personal compensation from Otsuka Pharmaceutical, Pfizer, Eli Lilly, Mitsubishi Tanabe Pharma, Mochida Pharmaceutical, Meiji Seika Pharma, Janssen Pharmaceutical, Takeda Pharmaceutical, MSD, Dainippon Sumitomo Pharma, and Eisai, and grants from Otsuka Pharmaceutical, Eli Lilly, Eisai, Shionogi, Takeda Pharmaceutical, MSD, and Pfizer. Additionally, Masahiko Ichiki is a member of the advisory board of Meiji Seika Pharma. Ichiro Kusumi has received personal compensation from Astellas, Chugai Pharmaceutical, Janssen Pharmaceutical, and Nippon Chemiphar, grants from AbbVie GK, Asahi 
Kasei Pharma, and Boehringer Ingelheim, and grants and personal compensation from Daiichi Sankyo, Dainippon Sumitomo Pharma, Eisai, Eli Lilly, GlaxoSmithKline, Kyowa Hakko Kirin, Meiji Seika Pharma, MSD, Novartis Pharma, Ono Pharmaceutical, Otsuka Pharmaceutical, Pfizer, Takeda Pharmaceutical, Mitsubishi Tanabe Pharma, Shionogi, and Yoshitomiyakuhin. Takeshi Inoue has received personal compensation from GlaxoSmithKline, Mochida Pharmaceutical, Asahi Kasei Pharma, Shionogi, Dainippon Sumitomo Pharma, and Takeda Pharmaceutical, grants from Astellas, and grants and personal compensation from Otsuka Pharmaceutical, Eli Lilly, Eisai, Mitsubishi Tanabe Pharma, Pfizer, AbbVie GK, MSD, Yoshitomiyakuhin, and Meiji Seika Pharma. Takeshi Inoue is also a member of the advisory boards of GlaxoSmithKline, Pfizer, Eli Lilly, Mochida Pharmaceutical, and Mitsubishi Tanabe Pharma. The authors report no other actual or potential conflicts of interest related this work.

\section{References}

1. Caspi A, Sugden K, Moffitt TE, et al. Influence of life stress on depression: moderation by a polymorphism in the 5-HTT gene. Science. 2003; 301(5631):386-389.

2. Kessler RC, Magee WJ. Childhood adversities and adult depression: basic patterns of association in a US national survey. Psychol Med. 1993; 23(3):679-690.

3. Weich S, Patterson J, Shaw R, Stewart-Brown S. Family relationships in childhood and common psychiatric disorders in later life: systematic review of prospective studies. Br J Psychiatry. 2009;194(5): 392-398.

4. Wise LA, Zierler S, Krieger N, Harlow BL. Adult onset of major depressive disorder in relation to early life violent victimisation: a case-control study. Lancet. 2001;358(9285):881-887.

5. Matsumoto S, Akiyama T, Tsuda H, et al. Reliability and validity of TEMPS-A in a Japanese non-clinical population: application to unipolar and bipolar depressives. $J$ Affect Disord. 2005;85(1-2):85-92.

6. Nakai Y, Inoue T, Toda H, et al. The influence of childhood abuse, adult stressful life events and temperaments on depressive symptoms in the nonclinical general adult population. J Affect Disord. 2014;158 101-107.

7. Nakai $\mathrm{Y}$, Inoue $\mathrm{T}$, Chen $\mathrm{C}$, et al. The moderator effects of affective temperaments, childhood abuse and adult stressful life events on depressive symptoms in the nonclinical general adult population. J Affect Disord. 2015;187:203-210.

8. Boyce P, Parker G. Development of a scale to measure interpersonal sensitivity. Aust N Z J Psychiatry. 1989;23(3):341-351.

9. Boyce P, Hickie I, Parker G, Mitchell P, Wilhelm K, Brodaty H. Interpersonal sensitivity and the one-year outcome of a depressive episode Aust N Z J Psychiatry. 1992;26(2):156-161.

10. Sakado K, Kuwabara H, Sato T, Uehara T, Sakado M, Someya T. The relationship between personality, dysfunctional parenting in childhood, and lifetime depression in a sample of employed Japanese adults. $J$ Affect Disord. 2000;60(1):47-51.

11. Boyce P, Parker G, Barnett B, Cooney M, Smith F. Personality as a vulnerability factor to depression. Br J Psychiatry. 1991;159:106-114.

12. Wilhelm K, Boyce P, Brownhill S. The relationship between interpersonal sensitivity, anxiety disorders and major depression. $J$ Affect Disord. 2004;79(1-3):33-41.

13. Okumura Y, Higuchi T. Cost of depression among adults in Japan. Prim Care Companion CNS Disord. 2011;13(3):e1-e9.
14. Girardi P, Pompili M, Innamorati M, et al. Duloxetine in acute major depression: review of comparisons to placebo and standard antidepressants using dissimilar methods. Hum Psychopharmacol. 2009;24(3): 177-190.

15. Nanni V, Uher R, Danese A. Childhood maltreatment predicts unfavorable course of illness and treatment outcome in depression: a metaanalysis. Am J Psychiatry. 2012;169(2):141-151.

16. Tomaszewska W, Peselow ED, Barouche F, Fieve RR. Antecedent life events, social supports and response to antidepressants in depressed patients. Acta Psychiatr Scand. 1996;94(5):352-357.

17. Bock C, Bukh JD, Vinberg M, Gether U, Kessing LV. The influence of comorbid personality disorder and neuroticism on treatment outcome in first episode depression. Psychopathology. 2010;43(3):197-204.

18. Pompili M, Iliceto P, Innamorati M, et al. Suicide risk and personality traits in physically and/or sexually abused acute psychiatric inpatients: a preliminary study. Psychol Rep. 2009;105(2):554-568.

19. Luty SE, Joyce PR, Mulder RT, Sullivan PF, McKenzie JM. The interpersonal sensitivity measure in depression: associations with temperament and character. J Affect Disord. 2002;70(3):307-312.

20. Otani K, Suzuki A, Ishii G, Matsumoto Y, Kamata M. Relationship of interpersonal sensitivity with dimensions of the Temperament and Character Inventory in healthy subjects. Compr Psychiatry. 2008;49(2): 184-187.

21. Akiskal HS, Akiskal KK, Haykal RF, Manning JS, Connor PD. TEMPS-A: progress towards validation of a self-rated clinical version of the Temperament Evaluation of the Memphis, Pisa, Paris, and San Diego Autoquestionnaire. J Affect Disord. 2005;85(1-2):3-16.

22. Kanai Y, Takaesu Y, Nakai Y, et al. The influence of childhood abuse, adult life events, and affective temperaments on the well-being of the general, nonclinical adult population. Neuropsychiatr Dis Treat. 2016;12: 823-832.

23. Muramatsu K, Miyaoka H, Kamijima K, et al. The patient health questionnaire, Japanese version: validity according to the miniinternational neuropsychiatric interview-plus. Psychol Rep. 2007; 101(3 Pt 1):952-960.

24. Akiyama T, Tsuda H, Matsumoto S, Kawamura Y, Miyake Y. [Cyclothymia and typus melancholicus: empirical study on personality character of mood disorder]. Seishin Shinkeigaku Zasshi. 2003;105(5):533-543. Japanese [with English abstract].

25. Sanders B, Becker-Lausen E. The measurement of psychological maltreatment: early data on the Child Abuse and Trauma Scale. Child Abuse Negl. 1995;19(3):315-323.

26. Tanabe H, Ozawa S, Goto K. [Psychometric properties of the Japanese version of the Child Abuse and Trauma Scale (CATS)]. Poster presented at: The 9th Annual Meeting of the Japanese Society for Traumatic Stress Studies; March 6, 2010; Kobe. Japanese.

27. Kuwabara H, Sakado K, Uehara T, Sakado M, Sato T, Someya T. [The Japanese version of Interpersonal Sensitivity Measure: its reliability and validity]. Arch Psychiatr Diagn Clin Eval. 1999;10:333-341. Japanese [with English abstract].

28. Schermelleh-Engel K, Moosbrugger H, Müller H. Evaluating the fit of structural equation models: tests of significance and descriptive goodness-of-fit measures. MPR Online. 2003;8(2):23-74.

29. Kroenke K, Spitzer RL, Williams JB. The PHQ-9: validity of a brief depression severity measure. J Gen Intern Med. 2001;16(9):606-613.

30. Akiskal HS, Mendlowicz MV, Jean-Louis G, et al. TEMPS-A: validation of a short version of a self-rated instrument designed to measure variations in temperament. $J$ Affect Disord. 2005;85(1-2):45-52.

31. Rovai L, Maremmani AG, Rugani F, et al. Do Akiskal \& Mallya's affective temperaments belong to the domain of pathology or to that of normality? Eur Rev Med Pharmacol Sci. 2013;17(15):2065-2079.

32. Engel-Yeger B, Muzio C, Rinosi G, et al. Extreme sensory processing patterns and their relation with clinical conditions among individuals with major affective disorders. Psychiatry Res. 2016;236: $112-118$.

33. McCauley J, Kern DE, Kolodner K, et al. Clinical characteristics of women with a history of childhood abuse: unhealed wounds. JAMA. 1997;277(17):1362-1368. 
34. Suzuki A, Matsumoto Y, Shibuya N, et al. Interaction effect between the BDNF Val66Met polymorphism and parental rearing for interpersonal sensitivity in healthy subjects. Psychiatry Res. 2012;200(2-3): 945-948.

35. Boyce P, Hickie I, Parker G. Parents, partners or personality? Risk factors for post-natal depression. J Affect Disord. 1991;21(4):245-255.

36. Perugi G, Toni C, Maremmani I, et al. The influence of affective temperaments and psychopathological traits on the definition of bipolar disorder subtypes: a study on bipolar I Italian national sample. J Affect Disord. 2012;136(1-2):e41-e49.
37. Robertson M, Rushton P, Wurm C. Interpersonal psychotherapy: an overview. Psychother Aust. 2008;14(3):46-54.

38. Brewin CR, Andrews B, Gotlib IH. Psychopathology and early experience: a reappraisal of retrospective reports. Psychol Bull. 1993; 113(1):82-98.

39. Toda $\mathrm{H}$, Inoue $\mathrm{T}$, Tsunoda $\mathrm{T}$, et al. The structural equation analysis of childhood abuse, adult stressful life events, and temperaments in major depressive disorders and their influence on refractoriness. Neuropsychiatr Dis Treat. 2015;11:2079-2090.

\section{Publish your work in this journal}

Neuropsychiatric Disease and Treatment is an international, peerreviewed journal of clinical therapeutics and pharmacology focusing on concise rapid reporting of clinical or pre-clinical studies on a range of neuropsychiatric and neurological disorders. This journal is indexed on PubMed Central, the 'PsycINFO' database and CAS, and is the official journal of The International Neuropsychiatric Association (INA). The manuscript management system is completely online and includes a very quick and fair peer-review system, which is all easy to use. Visit http://www.dovepress.com/testimonials.php to read real quotes from published authors.

Submit your manuscript here: http://www.dovepress.com/neuropsychiatric-disease-and-treatment-journal 